\section{Looking for CDKN1C enhancers}

European Journal of Human Genetics (2014) 22, 442-443; doi:10.1038/ejhg.2013.234; published online 16 October 2013

Gurrieri et al $^{1}$ recently described a familial case with mild BeckwithWiedemann Syndrome (BWS) and mild Silver-Russell Syndrome (SRS) phenotypes due to opposite parental origin of a $198 \mathrm{~kb}$ deletion on chromosome 11p15.5. Our group identified another deletion (of $60 \mathrm{~kb}$ ) that eliminates a subfragment of the region missing in the $198 \mathrm{~kb}$ deletion on the paternal chromosome, but is associated with severe Intra-Uterine Growth Restriction (IUGR). ${ }^{2}$ Careful analysis of the epigenetic marks reported by the ENCODE Project for this genomic region provides insights into the location of the cis-acting regulatory elements of the $11 \mathrm{p} 15.5$ region and a possible explanation for the phenotypic differences between the two clinical cases.
Altered expression of imprinted genes on chromosome 11p15.5 is associated with the growth disorders BWS (MIM \#130650) and SRS (MIM \#180860). ${ }^{3}$ The $11 \mathrm{p} 15.5$ imprinted gene cluster is divided into two distinct domains, each including an own imprinting control region (ICR). BWS and SRS are characterized by opposite growth phenotypes, and DNA methylation defects in the ICRs account for $60 \%$ of their molecular abnormalities. However, in a significant number of patients $(>30 \%)$ no molecular defect has been identified yet.

The centromeric domain of the cluster is $800 \mathrm{~kb}$ long and includes several imprinted genes. Its ICR (ICR2) is located within KCNQ1 intron 10 , it is methylated on the maternal chromosome and encompasses the promoter for the non-coding RNA KCNQ1OT1 (antisense to KCNQ1). Transcription of KCNQ1OT1 on the non-methylated paternal chromosome results in cis bi-directional silencing of the flanking imprinted genes. Among these genes, there is CDKN1C that encodes a cyclin-dependent kinase inhibitor and has growth suppressing properties. Decreased CDKN1C activity due to the loss of ICR2 methylation and maternal KCNQ1OT1 activation has been associated with BWS, ${ }^{4}$ whereas increased $C D K N 1 C$ activity due to maternal $11 \mathrm{p} 15.5$ duplication has been involved in SRS. ${ }^{5}$

John et al $\mathrm{s}^{6}$ work in the mouse indicates that, in addition to ICR2, $C d k n 1 c$ is regulated by distant enhancers likely located within the Kcnq1 gene. Enhancer sequences are identified as conserved non-coding regions $(>100 \mathrm{bp}$, which are $>70 \%$ identical between human and

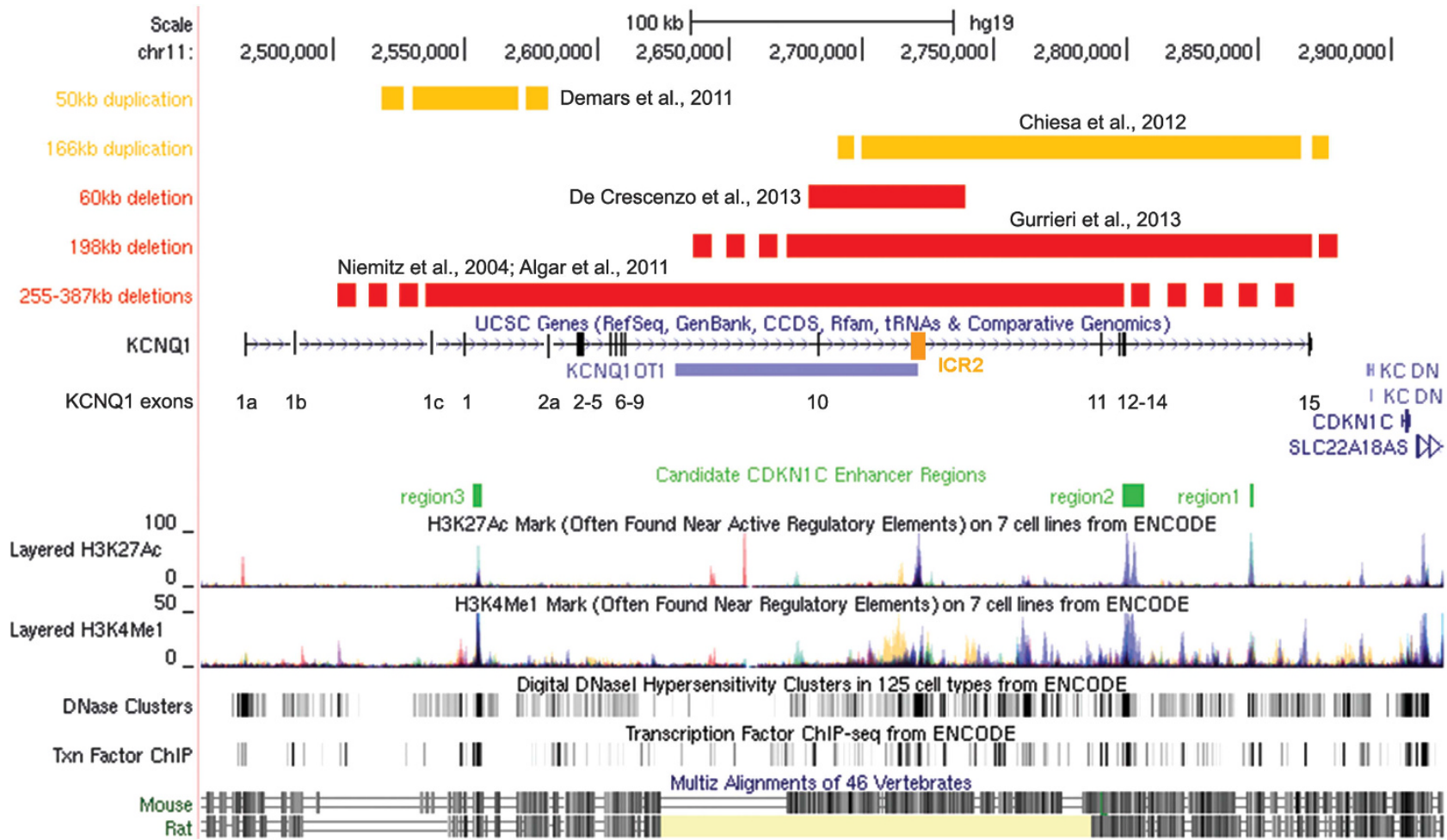

Figure 1 Identification of candidate $C D K N 1 C$ enhancer regions through integration of data of chromosome imbalances, chromatin features and sequence conservation at 11 p15.5. The image shows an UCSC Genome Browser screen shot including the most relevant information used to locate the putative enhancers. Three deletions associated with BWS/SRS or severe IUGR $1,2,10,11$ and two duplications associated with BWS 12,13 are indicated by red and yellow lines, respectively. Dots indicate undefined borders of duplications/deletions. The exons of the Kcnq1 gene (NM_000218) are numbered. ${ }^{15}$ ICR2 in KCNQ1 intron 10 is shown as an orange box. Green boxes indicate the candidate enhancers (region 1, chr11: 2846000-2849000; region 2, chr11: 2 798000-2807 000; region 3, chr11: $2552500-2556500$ - GRCh37/hg19, February 2009 assembly). No sequence information is available in the rat genome (rn4, November 2004) for the region corresponding to human chr11: 2622 000-2 788000. KCDN, KCDNQ1DN. Note that regions 1-3 correspond to evolutionary conserved intronic regions that are enriched in H3K27Ac, H3K4Me1, DNase hypersensitive sites and transcription factors binding as reported by ENCODE (http://genome.ucsc.edu Chr11: 2450000-2920000; GRCh37/hg19, February 2009 assembly). 
rodents) that are enriched in specific histone modifications (ie, H3K27acetylation, H3K4monomethylation), DNase hypersensitive sites and transcription factor binding. ${ }^{7,8}$ Based on the conservation between human, mouse and rat genomes ${ }^{9}$ and the epigenetic marks deposited by the ENCODE project, ${ }^{8}$ we found three sequences meeting the features of enhancers within the KCNQ1 gene region (Figure 1, green boxes and Supplementary Figure 1). Two of these sequences are located within KCNQ1 last intron (regions 1 and 2) and one in intron 1 immediately $3^{\prime}$ to the exon 1 boundary (region 3 ). Some of the characteristic features of the enhancers are also present in the ICR2/KCNQ1OT1 promoter which, however, also displays H3K4Me3 marks (not shown).

In human, rare chromosome imbalances involving ICR2 and different portions of the KCNQ1 gene have been reported associated with BWS/SRS phenotypes and severe IUGR (Figure 1). A deletion of 255-387 kb removing most of KCNQ1 (from intron $1 \mathrm{~b}$ to intron 14) was first described. ${ }^{10,11}$ This deletion results in CDKN1C downregulation and BWS when maternally inherited, but in normal phenotype on paternal transmission. More recently, a $198 \mathrm{~kb}$ deletion extending from KCNQ1 intron 9 to exon 15 was found associated with mild BWS when maternally inherited and mild SRS when paternally inherited. ${ }^{1}$ Finally, a paternal deletion of $60 \mathrm{~kb}$ resulting in KCNQ1OT1 silencing and CDKN1C activation was associated with severe and recurrent IUGR. ${ }^{2}$ The existence of multiple CDKN1C enhancers was originally proposed to explain the phenotypes of the $255-387 \mathrm{~kb}$ and $198 \mathrm{~kb}$ deletions. ${ }^{1,11}$ To reconcile the phenotypes of all three deletions, we now propose that these enhancers are all (or mostly) located within the $255-387 \mathrm{~kb}$ deletion, in part within the $198 \mathrm{~kb}$ deletion, but none within the $60 \mathrm{~kb}$ deletion (Figure 1). So, by eliminating ICR2 but keeping the enhancers on the paternal chromosome, the $60 \mathrm{~kb}$ deletion would abolish the silencing effect of the KCNQ1OT1 RNA and lead to CDKN1C activation and severe growth restriction. In contrast, by eliminating one or more enhancers, the larger deletions would result in weaker (198 kb deletion) or no activation (255-387 kb deletion) of the paternal CDKN1C. Interestingly, all the candidate enhancer sequences indicated by the ENCODE data lie outside the $60 \mathrm{~kb}$ deletion and likely within the $255-387 \mathrm{~kb}$ deletion, but only two of them are within the $198 \mathrm{~kb}$ deletion, thus meeting the characteristics proposed for the CDKN1C enhancers. These regions may also be involved in imprinting control, as they are included in two maternal duplications (Figure 1) associated with ICR2 hypomethylation and BWS. ${ }^{12,13}$ Intriguingly, region 3 also harbors strong binding sites for CTCF (Supplementary Figure 1), which have been shown to interact with other CTCF binding sequences near CDKN1C, possibly forming higher-order chromatin structures or insulators that may be disrupted by the duplication and result in ICR2 imprinting alteration. ${ }^{14}$

In conclusion, by integrating information on chromosome rearrangements and chromatin features, we have identified three sequences meeting the features predicted for CDKN1C enhancers that help explain the phenotypes associated with different $11 \mathrm{p} 15.5$ deletions. Further experimental work in animal models (transgenic enhancer assays) and in the patients (mutation screening) will possibly confirm their functional role.

\section{CONFLICT OF INTEREST}

The authors declare no conflict of interest.

\section{ACKNOWLEDGEMENTS}

This study was supported by Telethon-Italia grant no. GGP11122, and Associazione Italiana Ricerca sul Cancro (to AR).

Flavia Cerrato ${ }^{1}$, Agostina De Crescenzo ${ }^{1}$ and Andrea Riccio ${ }^{\star 1,2}$ ${ }^{1}$ Department of Environmental, Biological and Pharmaceutical Sciences and Technologies, Second University of Naples, Caserta, Italy;

${ }^{2}$ Institute of Genetics and Biophysics A.Buzzati-Traverso, CNR, Naples, Italy E-mail: andrea.riccio@unina2.it

1 Gurrieri F, Zollino M, Oliva A et al: Mild Beckwith-Wiedemann and severe long-QT syndrome due to deletion of the imprinting center 2 on chromosome 11p. Eur J Hum Genet 2013; 21: 965-969.

2 De Crescenzo A, Sparago A, Cerrato $F$ et al: Paternal deletion of the 11p15.5 centromeric-imprinting control region is associated with alteration of imprinted gene expression and recurrent severe intrauterine growth restriction. J Med Genet 2012; 50: 99-103.

3 Demars J, Gicquel C: Epigenetic and genetic disturbance of the imprinted 11p15 region in Beckwith-Wiedemann and Silver-Russell syndromes. Clin Genet 2012; 81: 350-361.

4 Diaz-Meyer N, Day CD, Khatod K et al: Silencing of CDKN1C (p57KIP2) is associated with hypomethylation at KvDMR1 in Beckwith-Wiedemann syndrome. J Med Genet 2003; 40: 797-801.

5 Schönherr N, Meyer E, Roos A, Schmidt A, Wollmann HA, Eggermann T: The centromeric $11 \mathrm{p} 15$ imprinting centre is also involved in Silver-Russell syndrome. J Med Genet 2007; 44: 59-63.

6 John RM, Ainscough JF, Barton SC, Surani MA: Distant cis-elements regulate imprinted expression of the mouse p57( Kip2) (Cdkn1c) gene: implications for the human disorder, Beckwith-Wiedemann syndrome. Hum Mol Genet 2001; 15: 1601-1609.

7 Frazer KA, Pachter L, Poliakov A, Rubin EM, Dubchak I: VISTA: computational tools for comparative genomics. Nucleic Acids Res 2004; 32(Web Server issue): W273-W279.

8 The ENCODE Project Consortium. An integrated encyclopedia of DNA elements in the human genome. Nature 2012; 489: 57-74.

9 Blanchette M, Kent WJ, Riemer C: Aligning multiple genomic sequences with the threaded blockset aligner. Genome Res 2004; 14: 708-715.

10 Niemitz EL, DeBaun MR, Fallon J et al: Microdeletion of LIT1 in familial BeckwithWiedemann syndrome. Am J Hum Genet 2004; 75: 844-849.

11 Algar E, Dagar V, Sebaj M, Pachter N: An 11p15 imprinting centre region 2 deletion in a family with Beckwith Wiedemann syndrome provides insights into imprinting control at CDKN1C. PLoS One 2011; 6: e29034.

12 Chiesa N, De Crescenzo A, Mishra $\mathrm{K}$ et al: The KCNQ1OT1 imprinting control region and non-coding RNA: new properties derived from the study of BeckwithWiedemann syndrome and Silver-Russell syndrome cases. Hum Mol Genet 2012; 21 : 10-25.

13 Demars J, Shmela ME, Rossignol S et al: New insights into the pathogenesis of Beckwith-Wiedemann and Silver-Russell syndromes: contribution of small copy number variations to $11 \mathrm{p} 15$ imprinting defects. Hum Mutat 2011; 32: 1171-1182.

14 Handoko L, Xu H, Li G et al: CTCF-mediated functional chromatin interactome in pluripotent cells. Nat Genet 2011; 43: 630-638.

15 Neyroud N, Richard P, Vignier N et al: Genomic organization of the KCNQ1 K+ channel gene and identification of C-terminal mutations in the long-QT syndrome. Circ Res 1999; 84: 290-297.

Supplementary Information accompanies this paper on European Journal of Human Genetics website (http://www.nature.com/ejhg) 